\title{
Dynamics of Infected Predator-Prey System with Nonlinear Incidence Rate and Prey in Refuge
}

\author{
Adin Lazuardy Firdiansyah \\ STAI Muhammadiyah Probolinggo, adin.lazuardy@gmail.com
}

doi: https://doi.org/10.15642/mantik.2020.6.2.123-134

\begin{abstract}
Abstrak. Model mangsa-pemangsa dengan laju kejadian nonlinear dan perlindungan dimunculkan untuk menggambarkan perubahan prilaku pada mangsa yang sehat ketika jumlah mangsa yang terinfeksi meningkat, sedangkan pemangsa dapat memakan mangsa dengan mengakses perlindungan pada mangsa. Oleh karena itu, analisis dinamik model mangsa-pemangsa dengan penyebaran penyakit yang dinotasikan dengan laju kejadian nonlinear dan perlindungan mangsa dibahas pada penilitian ini. Hasil analisis ditemukan bahwa ada delapan titik kesetimbangan, dimana semua titik kesetimbangan tersebut stabil asimtotik secara lokal. Selanjutnya, perlindungan mangsa juga dipertimbangkan pada penelitian ini. Perlindungan mangsa memiliki pengaruh penting pada model. Hal ini ditunjukkan dengan adanya perlindungan mangsa yang dapat mencegah kepunahan pada populasi mangsa. Pada bagian akhir, simulasi numerik dilakukan untuk mengilustrasikan hasil analisis yang diperoleh. Untuk penelitian berikutnya, model mangsa-pemangsa dapat diinvestigasi efek pemanenanya untuk kedua populasi.
\end{abstract}

Kata kunci: Model mangsa-pemangsa; Laju kejadian nonlinear; Perlindungan; Kestabilan

\begin{abstract}
A predator-prey system with nonlinear incidence rate and refuging in prey is proposed to describe behavior change of certain infected diseases on healthy prey when the number of infected prey is getting large, while predator can predate prey by accessing refuging in prey. Therefore, this paper discusses the dynamics behavior predator-prey model with the spread of infected disease that is denoted by nonlinear incidence rate and adding prey refuge. We find the existence of eight nonnegative equilibrium in the model, which their local stability has been determined. Furthermore, we also observe the prey refuge properties in the model. We find that prey refuge can prevent extinction in prey populations. In the end, some numerical solutions are carried out to illustrate our analytic results. For future work, we can investigate the harvesting effect in both populations, which is disease control in the predator-prey model with the spread of infected disease.
\end{abstract}

Keywords: Predator-prey system; Nonlinear incidence rate; Refuge; Stability

How to cite: A. L. Firdiansyah, "Dynamics of Infected Predator-Prey System with Nonlinear Incidence Rate and Prey in Refuge", J. Mat. Mantik, vol. 6, no. 2, pp. 123-134, October 2020. 


\section{Introduction}

After Lotka and Volterra proposed a mathematical model that could represent the interactions of predators and prey, many researchers were interested to study the model. Similarly, Kermack and McKendrick [1] also proposed a SIR (Susceptible-InfectiousRecovered) epidemic model that represented the infected disease transmission. After that, Anderson and May [2] for the first time proposed the eco-epidemiology model and investigated invasion, persistence, and spread of infected disease in the model. Since then, many researchers have studied the eco-epidemiology model. Generally speaking, the ecoepidemiology model is divided into three cases. The first case is an only infected disease in prey as in [3]-[5]. The second case is an only infected disease in predator as in [6]. Meanwhile, the third case is an infected disease in both populations as in [7]-[9].

In the eco-epidemiology model, there is a basic component that can represent the spread of infected disease. Generally, it is denoted by the simple mass incidence rate, $\beta S I$, with $\beta$ means the infection rate. This incidence rate shows that the transmission disease with rate $\beta S I$, i.e. when the transmission disease increases significantly, then the number of infected population increases too [1]. In the model [3]-[8], the authors assume the simple mass incidence rate to represent the transmission disease in the model.

In several cases, the simple mass incidence rate doesn't produce appropriate results. When the amount of infected population increases significantly, the susceptible population tend to change their behavior to reduce contact with the infected population. Therefore, Capasso and Serio [10] proposed the saturated incidence rate to describe the transmission disease. As done in [9], they investigated the local stability where the transmission disease in the eco-epidemiology model followed the saturated incidence rate. However, there is a nonlinear incidence rate that is suggested by [11]. They introduced nonlinear incidence rate, $\frac{\beta I^{p}}{1+\alpha I^{q}}\left(p, q, \alpha, \beta\right.$ were positive constants), where $\beta I^{p}$ meant infection rate and $\frac{1}{1+\alpha I^{q}}$ meant inhibition rate from the behavior change on healthy population when infected population was getting large. Many researcher uses this incidence rate by giving $p, q, \alpha$ makes different values as in [12]-[14]. This becomes more rational because it includes the effect of tightness from infective individuals [12].

Recently, the effect of disease in the eco-epidemiology model has become an important topic for many researchers. However, other components that can influence the dynamic of species interactions is the Allee effect, habitat complexity, harvesting, and prey refuge. For the prey refuge effect, theoretical research provides a conclusion that it can influence stabilizing and destabilizing the predator-prey model and can avoid extinction in prey [15]-[17]. It can make the predator-prey model to form more realistic.

Motivated by previous research, we modify a model from [9] by including nonlinear incidence rate and adding the effect of prey refuge. At this time, several similar models have emerged, but the latest distinctive feature of our model is the inclusion of transmission disease in both populations and also the inclusion of prey refuge properties of the prey population. Incorporation of prey refuge gives a factor which can be accessed by predator populations. Under this adding effect, our model is more realistic and differs from the previous eco-epidemiology model. The model is analyzed to determine the local stability of its equilibrium points. Moreover, several simulations are given to demonstrate the results of our analysis.

\section{Methods}

The method used for this research is the literature study which studies some previous research. It's used to modify a model from [9]. The steps to solve this research are below: a. Reviewing and studying the predator-prey model from previous research. 
b. Modifying the predator-prey model by including nonlinear incidence rate and adding the effect of prey refuge.

c. Analyzing the existence of equilibrium points and local stability in the modified model.

d. Performing numerical simulation by using the Runge-Kutta $4^{\text {th }}$ order method as a numerical method to support our analysis results.

e. Making a conclusion based on the analysis results.

\section{Result and Discussion}

\subsection{The Formulation Model}

Our mathematical model contains two populations, namely the prey population and predator population. At time $t$, susceptible and infected prey are denoted by $S(t)$ and $I(t)$, respectively. Meanwhile, susceptible and infected predator are denoted by $Y_{S}(t)$ and $Y_{I}(t)$, respectively. The following is several basic assumptions for our mathematical model:

a. In the absence of predation and disease, the prey grows according to logistics with a growth rate $r(r>0)$ and carrying capacity $K(K>0)$.

b. Only one population that can reproduce, namely susceptible prey.

c. The prey has one infection source like viruses or other sources. Meanwhile, the predator can be infected due they eat the infected prey.

d. The transmission disease follows the nonlinear incidence rate $\frac{\beta S I}{1+I}$, where $\beta I$ means infection rate and $\frac{1}{1+I}$ means inhibition rate from behavior change on healthy prey, when infected prey is getting large. Meanwhile, the transmission disease in predator follows the simple mass incidence rate $\left(\gamma Y_{S} Y_{I}\right)$, where $\gamma$ expressed infection rate.

e. Both of infected prey and predator isn't recovered and no get immune.

f. The infected predator can't predate healthy prey. Both of infected predator and susceptible predator can eat infected prey because it is easy to be predated by them. But, the ability to catch from an infected predator is lower than susceptible predator.

g. All species have natural death rates and death rates due to infection.

h. The functional response for predation of predator is the Lotka-Volterra type.

i. The refuge protection can be denoted with $\left(1-m_{3}\right) S$ for susceptible prey and $\left(1-m_{4}\right) I$ for infected prey, where $m_{3}, m_{4} \in(0,1]$ and healthy prey is more agile than infected prey.

The details of the model structure are shown in the schematic flow diagram as in figure 1 . From the flow chart in figure 1, the mathematical model is presented as follows:

$$
\begin{aligned}
\frac{d S}{d t} & =r S\left(1-\frac{S+I}{K}\right)-\frac{\beta S I}{1+I}-p_{1}\left(1-m_{3}\right) S Y_{S}-a_{1} S, \\
\frac{d I}{d t} & =\frac{\beta S I}{1+I}-p_{2}\left(1-m_{4}\right) I Y_{S}-p_{3}\left(1-m_{4}\right) I Y_{I}-a_{2} I, \\
\frac{d Y_{S}}{d t} & =a_{3}\left(1-m_{3}\right) S Y_{S}+a_{4}\left(1-m_{4}\right) I Y_{S}-\gamma Y_{S} Y_{I}-d_{3} Y_{S}, \\
\frac{d Y_{I}}{d t} & =a_{5}\left(1-m_{4}\right) I Y_{I}+\gamma Y_{S} Y_{I}-a_{6} Y_{I} .
\end{aligned}
$$

with the initial condition as $S(0)>0, I(0)>0, Y_{S}(0)>0, Y_{I}(0)>0$. All parameters with their biological meaning are given as follows: $a_{1}=m_{1}+d_{1}, a_{2}=m_{2}+d_{2}+c, a_{3}=$ $p_{1} q_{1}, a 4=p_{2} q_{2}, a_{5}=p_{3} q_{3}$, and $a_{6}=d_{4}+d_{5}$ where $m_{1}$ and $m_{2}$ are migration rates for susceptible prey and infected prey, respectively. $p_{1}\left(p_{2}\right)$ and $p_{3}$ are predation rate from healthy predator to healthy prey (to infected prey) and predation rate from infected predator to infected prey, respectively. $q_{1}\left(q_{2}\right)$ and $q_{3}$ are conversion rate from healthy prey into healthy predator (infected predator) and conversion rate from infected prey into an infected 
predator. $d_{1}\left(d_{2}\right)$ and $d_{3}\left(d_{4}\right)$ are natural death from susceptible prey (infected prey) and natural death from a susceptible predator (infected predator), respectively. $c\left(d_{5}\right)$ is the death rate from infected prey (from an infected predator) due to infection from disease. We assume that all parameters are positive values.

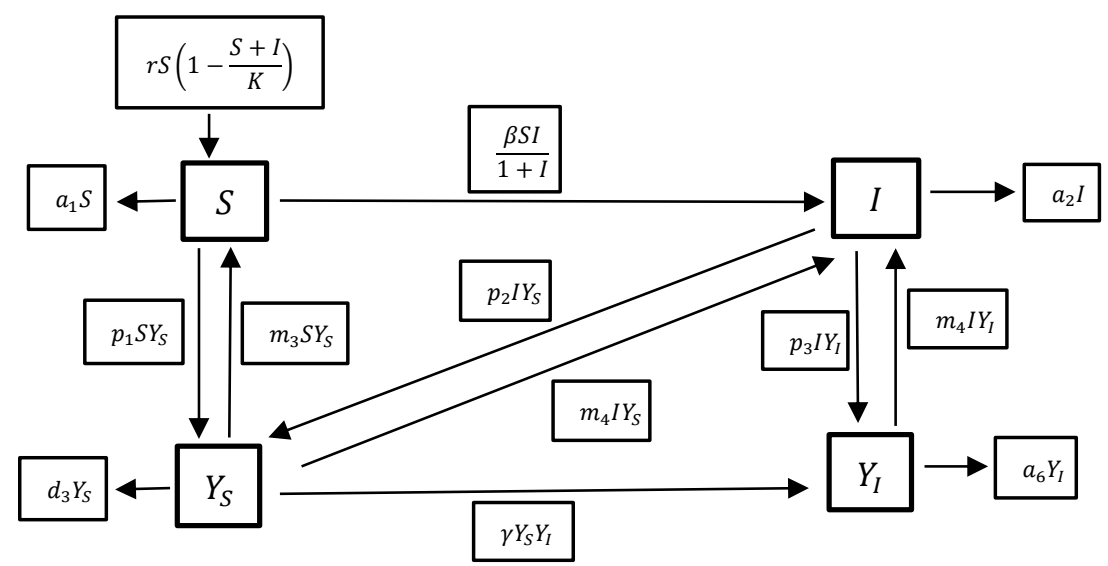

Figure 1. Schematic diagram of the model

\subsection{Equilibrium Point of Mathematical Model}

We set the right-hand sides equal to zero. Thus, we get possible equilibriums:

a. The trivial equilibrium point is $E_{0}(0,0,0,0)$. This point always exists.

b. The axial equilibrium point is $E_{1}\left(S^{(1)}, 0,0,0\right)$, where $S^{(1)}=\frac{K\left(r-a_{1}\right)}{r}$. It will exist when $r>a_{1}$.

c. $E_{2}\left(S^{(2)}, I^{(2)}, 0,0\right)$ is the predator-free equilibrium point, where

$$
S^{(2)}=\frac{\left(1+I^{(2)}\right)\left(r K-r I^{(2)}-K a_{1}\right)-K \beta I^{(2)}}{r\left(1+I^{(2)}\right)}
$$

and $I^{(2)}$ is the positive root of quadratic equations $A_{0}\left(I^{(2)}\right)^{2}+A_{1} I^{(2)}+A_{2}=0$, where $A_{0}=r\left(a_{2}+\beta\right), A_{1}=2 r a_{2}-\beta r K+\beta r+\beta a_{1} K+\beta^{2} K$, and $A_{2}=r a_{2}-$ $\beta r K+\beta a_{1} K$. The equilibrium $E_{2}$ will exist if $r \geq \frac{\beta a_{1} K}{\beta K-a_{2}}$.

d. $E_{3}\left(S^{(3)}, 0, Y_{S}^{(3)}, 0\right)$ is the disease-free equilibrium point, where

$$
S^{(3)}=\frac{d_{3}}{a_{3}\left(1-m_{3}\right)}
$$

and

$$
Y_{S}^{(3)}=\frac{K\left(r-a_{1}\right)-r S^{(3)}}{K p_{1}\left(1-m_{3}\right)}
$$

The equilibrium point $E_{3}$ will exist if $r>\frac{a_{1} a_{3} K\left(1-m_{3}\right)}{a_{3} K\left(1-m_{3}\right)-d_{3}}$.

e. $\quad E_{4}\left(S^{(4)}, \frac{a_{6}}{a_{5}\left(1-m_{4}\right)}, 0, Y_{I}^{(4)}\right)$ is the healthy predator-free equilibrium point, where 


$$
S^{(4)}=\frac{\left(a_{5}\left(1-m_{4}\right)+a_{6}\right)\left(p_{3}\left(1-m_{4}\right) Y_{I}^{(4)}+a_{2}\right)}{\beta a_{5}\left(1-m_{4}\right)}
$$

and

$$
Y_{I}^{(4)}=\frac{C_{1}-C_{2}}{r p_{3}\left(1-m_{4}\right)\left(a_{5}\left(1-m_{4}\right)+a_{6}\right)^{2}}
$$

with $C_{1}=K \beta a_{5}\left(1-m_{4}\right)\left(r-a_{1}\right)\left(a_{5}\left(1-m_{4}\right)+a_{6}\right)$ and $C_{2}=r a_{2}\left(a_{5}\left(1-m_{4}\right)+\right.$ $\left.a_{6}\right)^{2}+K \beta^{2} a_{5} a_{6}\left(1-m_{4}\right)+r \beta a_{6}\left(a_{5}\left(1-m_{4}\right)+a_{6}\right)$. This equilibrium exists when $C_{1}>C_{2}$ and $r>a_{1}$.

f. $\quad E_{5}\left(S^{(5)}, 0, \frac{a_{6}}{\gamma}, Y_{I}^{(5)}\right)$ is the infected prey-free equilibrium point, where

$$
S^{(5)}=\frac{K \gamma\left(r-a_{1}\right)-K p_{1} a_{6}\left(1-m_{3}\right)}{r \gamma}
$$

and

$$
Y_{I}^{(5)}=\frac{\left(1-m_{3}\right) a_{3} S^{(5)}-d_{3}}{\gamma} .
$$

It will exist if $r>\frac{K a_{3}\left(1-m_{3}\right)\left(\gamma a_{1}+p_{1} a_{6}\left(1-m_{3}\right)\right)}{\gamma\left(K a_{3}\left(1-m_{3}\right)-d_{3}\right)}$.

g. $\quad E_{6}\left(\frac{d_{3}-a_{4}\left(1-m_{4}\right) I^{(6)}}{a_{3}\left(1-m_{3}\right)}, I^{(6)}, Y_{S}^{(6)}, 0\right)$ is the infected predator-free equilibrium, where

$$
Y_{S}^{(6)}=\frac{\beta d_{3}-\beta a_{4}\left(1-m_{4}\right) I^{(6)}-a_{2} a_{3}\left(1-m_{3}\right)\left(1+I^{(6)}\right)}{p_{2} a_{3}\left(1-m_{3}\right)\left(1-m_{4}\right)\left(1+I^{(6)}\right)}
$$

and $I^{(6)}$ is the positive root of quadratic equations $D_{0}\left(I^{(6)}\right)^{2}+D_{1} I^{(6)}+D_{2}=0$ with

$$
\begin{aligned}
D_{0}= & r p_{2}\left(1-m_{4}\right)\left(a_{3}\left(1-m_{3}\right)-a_{4}\left(1-m_{4}\right)\right) \\
D_{1}= & K a_{3} p_{2}\left(1-m_{3}\right)\left(1-m_{4}\right)\left(\beta+a_{1}-r\right) \\
& +r p_{2}\left(1-m_{4}\right)\left(d_{3}+a_{3}\left(1-m_{3}\right)-a_{4}\left(1-m_{4}\right)\right) \\
& -K p_{1}\left(1-m_{3}\right)\left(a_{2} a_{3}\left(1-m_{3}\right)+\beta a_{4}\left(1-m_{4}\right)\right) \\
D_{2}= & r p_{2}\left(1-m_{4}\right)\left(d_{3}-K a_{3}\left(1-m_{3}\right)\right) \\
& +K a_{3}\left(1-m_{3}\right)\left(a_{1} p_{2}\left(1-m_{4}\right)-a_{2} p_{1}\left(1-m_{3}\right)\right)+K \beta p_{1} d_{3}\left(1-m_{3}\right) .
\end{aligned}
$$

This equilibrium will exist if $I^{(6)}<\frac{\beta d_{3}-a_{2} a_{3}\left(1-m_{3}\right)}{\beta a_{4}\left(1-m_{4}\right)+a_{2} a_{3}\left(1-m_{3}\right)}$.

h. $\quad E_{7}\left(S^{(7)}, I^{(7)}, \frac{a_{6}-a_{5}\left(1-m_{4}\right) I^{(7)}}{\gamma}, \frac{a_{3}\left(1-m_{3}\right) S^{(7)}+a_{4}\left(1-m_{4}\right) I^{(7)}-d_{3}}{\gamma}\right)$ is the interior equilibrium point, where $S^{(7)}=\frac{\left(1+I^{(7)}\right)\left(K r \gamma-a_{1} \gamma K-r \gamma I^{(7)}-K p_{1}\left(1-m_{3}\right)\left(a_{6}-a_{5}\left(1-m_{4}\right) I^{(7)}\right)\right)-K \gamma \beta I^{(7)}}{\gamma r\left(1+I^{(7)}\right)}$ and $I^{(7)}$ is the positive root of the cubic equation $Q_{0}\left(I^{(7)}\right)^{3}+Q_{1}\left(I^{(7)}\right)^{2}+Q_{2} I^{(7)}+Q_{3}=$ 0 , with

$$
Q_{0}=K a_{3} a_{5} p_{1} p_{3}\left(1-m_{3}\right)^{2}\left(1-m_{4}\right)^{2}-\gamma r a_{3} p_{3}\left(1-m_{3}\right)\left(1-m_{4}\right)
$$




$$
\begin{aligned}
Q_{1}= & +\gamma r a_{4} p_{3}\left(1-m_{4}\right)^{2}-\gamma r a_{5} p_{2}\left(1-m_{4}\right)^{2}, \\
& 2 \gamma r a_{4} p_{3}\left(1-m_{4}\right)^{2}-2 \gamma r a_{5} p_{2}\left(1-m_{4}\right)^{2}+\gamma r a_{6} p_{2}\left(1-m_{4}\right)+\gamma^{2} r a_{2} \\
& -\gamma r d_{3} p_{3}\left(1-m_{4}\right)+2 K a_{3} a_{5} p_{1} p_{3}\left(1-m_{3}\right)^{2}\left(1-m_{4}\right)^{2}+\beta \gamma^{2} r \\
& -K a_{3} a_{6} p_{1} p_{3}\left(1-m_{3}\right)^{2}\left(1-m_{4}\right)-K \gamma a_{1} a_{3} p_{3}\left(1-m_{3}\right)\left(1-m_{4}\right) \\
& +K \gamma r a_{3} p_{3}\left(1-m_{3}\right)\left(1-m_{4}\right)-K \beta \gamma a_{3} p_{3}\left(1-m_{3}\right)\left(1-m_{4}\right) \\
& -K \beta \gamma a_{5} p_{1}\left(1-m_{3}\right)\left(1-m_{4}\right)-2 \gamma r a_{3} p_{3}\left(1-m_{3}\right)\left(1-m_{4}\right), \\
Q_{2}= & \gamma r a_{4} p_{3}\left(1-m_{4}\right)^{2}-\gamma r a_{5} p_{2}\left(1-m_{4}\right)^{2}+2 \gamma r a_{6} p_{2}\left(1-m_{4}\right)+2 \gamma^{2} r a_{2} \\
& -2 \gamma r d_{3} p_{3}\left(1-m_{4}\right)+K a_{3} a_{5} p_{1} p_{3}\left(1-m_{3}\right)^{2}\left(1-m_{4}\right)^{2}+\beta \gamma^{2} r \\
& -2 K a_{3} a_{6} p_{1} p_{3}\left(1-m_{3}\right)^{2}\left(1-m_{4}\right)-2 K \gamma a_{1} a_{3} p_{3}\left(1-m_{3}\right)\left(1-m_{4}\right) \\
& +2 K \gamma r a_{3} p_{3}\left(1-m_{3}\right)\left(1-m_{4}\right)-K \beta \gamma a_{3} p_{3}\left(1-m_{3}\right)\left(1-m_{4}\right)+K \beta^{2} \gamma^{2} \\
& -K \beta \gamma a_{5} p_{1}\left(1-m_{3}\right)\left(1-m_{4}\right)+K \beta \gamma a_{6} p_{1}\left(1-m_{3}\right)-K \beta \gamma^{2} r+K \beta \gamma^{2} a_{1} \\
& -\gamma r a_{3} p_{3}\left(1-m_{3}\right)\left(1-m_{4}\right), \\
Q_{3}= & K \gamma r a_{3} p_{3}\left(1-m_{3}\right)\left(1-m_{4}\right)-K \gamma a_{1} a_{3} p_{3}\left(1-m_{3}\right)\left(1-m_{4}\right) \\
& -K a_{3} a_{6} p_{1} p_{3}\left(1-m_{3}\right)^{2}\left(1-m_{4}\right)+K \beta \gamma a_{6} p_{1}\left(1-m_{3}\right) \\
& +\gamma r a_{6} p_{2}\left(1-m_{4}\right)-\gamma r d_{3} p_{3}\left(1-m_{4}\right)+K \beta \gamma^{2} a_{1}-K \beta \gamma^{2} r+\gamma^{2} r a_{2} .
\end{aligned}
$$

The existing form of the positive root in the cubic equation can be determined by using Cardan's method as in [18].

\subsection{Stability Analysis}

To check the local stability of the equilibrium point, we have to determine the eigenvalues of the Jacobian matrix. Here, the Jacobian matrix at the equilibrium point $E^{*}\left(S^{*}, I^{*}, Y_{S}^{*}, Y_{I}^{*}\right)$ is as follows:

$$
J^{*}=\left[\begin{array}{cccc}
u_{11} & u_{12} & -p_{1}\left(1-m_{3}\right) S^{*} & 0 \\
\frac{\beta I^{*}}{1+I^{*}} & u_{22} & -p_{2}\left(1-m_{4}\right) I^{*} & -p_{3}\left(1-m_{4}\right) I^{*} \\
a_{3}\left(1-m_{3}\right) Y_{S}^{*} & a_{4}\left(1-m_{4}\right) Y_{S}^{*} & u_{33} & -\gamma Y_{S}^{*} \\
0 & a_{5}\left(1-m_{4}\right) Y_{I}^{*} & \gamma Y_{I}^{*} & u_{44}
\end{array}\right],
$$

where

$$
\begin{aligned}
& u_{11}==r\left(1-\frac{S^{*}+I^{*}}{K}\right)-\frac{r S^{*}}{K}-\frac{\beta I^{*}}{1+I^{*}}-p_{1}\left(1-m_{3}\right) Y_{S}^{*}-a_{1}, \\
& u_{12}=-\frac{r S^{*}}{K}-\frac{\beta S^{*}}{1+I^{*}}+\frac{\beta S^{*} I^{*}}{\left(1+I^{*}\right)^{2}} \\
& u_{22}=\frac{\beta S^{*}}{1+I^{*}}-\frac{\beta S^{*} I^{*}}{\left(1+I^{*}\right)^{2}}-p_{2}\left(1-m_{4}\right) Y_{S}^{*}-p_{3}\left(1-m_{4}\right) Y_{I}^{*}-a_{2}, \\
& u_{33}=a_{3}\left(1-m_{3}\right) S^{*}+a_{4}\left(1-m_{4}\right) I^{*}-\gamma Y_{I}^{*}-d_{3}, \\
& u_{44}=a_{5}\left(1-m_{4}\right) I^{*}+\gamma Y_{I}^{*}-a_{6} .
\end{aligned}
$$

The characteristic equation of $E_{0}$ is

$$
\left(r-a_{1}-\lambda\right)\left(-a_{2}-\lambda\right)\left(-d_{3}-\lambda\right)\left(-a_{6}-\lambda\right)=0,
$$

which has eigenvalues $\lambda_{1}=r-a_{1} ; \lambda_{2}=-a_{2} ; \lambda_{3}=-d_{3} ; \lambda_{4}=-a_{6}$. Thus, $E_{0}$ is locally asymptotically stable if only if $r<a_{1}$. On point $E_{1}$, the characteristic equation of $E_{1}$ is

$$
\left(a_{1}-r-\lambda\right)\left(g_{1}-\lambda\right)\left(g_{2}-\lambda\right)\left(-a_{6}-\lambda\right)=0,
$$

with 


$$
\begin{aligned}
& g_{1}=\frac{\beta K\left(r-a_{1}\right)}{r}-a_{2}, \\
& g_{2}=\frac{a_{3} K\left(1-m_{3}\right)\left(r-a_{1}\right)}{r}-d_{3} .
\end{aligned}
$$

This point has eigenvalues $\lambda_{1}=a_{1}-r ; \lambda_{2}=g_{1} ; \lambda_{3}=g_{2} ; \lambda_{4}=-a_{6}$. Thus, $E_{1}$ can be said the locally asymptotically stable if only if $a_{1}<r<\min \left\{\frac{\beta K a_{1}}{\beta K-a_{2}}, \frac{K a_{1} a_{3}\left(1-m_{3}\right)}{K a_{3}\left(1-m_{3}\right)-d_{3}}\right\}$, where $\beta K>a_{2}$ and $K a_{3}\left(1-m_{3}\right)>d_{3}$. On point $E_{2}$, the characteristic equation of $E_{2}$ is

$$
\left(\lambda^{2}-\left(h_{1}+h_{2}\right) \lambda+\left(h_{1} h_{2}-h_{3} h_{2}\right)\right)\left(h_{4}-\lambda\right)\left(h_{5}-\lambda\right)=0,
$$

with

$$
\begin{aligned}
h_{1} & =r\left(1-\frac{S^{(2)}+I^{(2)}}{K}\right)-\frac{r S^{(2)}}{K}-\frac{\beta I^{(2)}}{1+I^{(2)}}-a_{1}, \\
h_{2} & =\frac{\beta S^{(2)}}{1+I^{(2)}}-\frac{\beta S^{(2)} I^{(2)}}{\left(1+I^{(2)}\right)^{2}}-a_{2}, \\
h_{3} & =\frac{\beta I^{(2)}}{1+I^{(2)}}, \\
h_{4} & =a_{3}\left(1-m_{3}\right) S^{(2)}+a_{4}\left(1-m_{4}\right) I^{(2)}-d_{3}, \\
h_{5} & =a_{5}\left(1-m_{4}\right) I^{(2)}-a_{6} .
\end{aligned}
$$

Eigenvalues on this point $E_{2}$ is $\lambda_{1,2}=\frac{\left(h_{1}+h_{2}\right) \pm \sqrt{\left(h_{1}+h_{2}\right)^{2}-4\left(h_{1} h_{2}-h_{3} h_{2}\right)}}{2} ; \lambda_{3}=h_{4} ; \lambda_{4}=h_{5}$. Thus, the point $E_{2}$ can be said the locally asymptotically stable if only if $h_{1}+h_{2}<0$; $h_{1} h_{2}>h_{3} h_{2} ; a_{5}\left(1-m_{4}\right) I^{(2)}<a_{6} ; a_{3}\left(1-m_{3}\right) S^{(2)}+a_{4}\left(1-m_{4}\right) I^{(2)}<d_{3}$. On point $E_{3}$, we obtain the characteristic equation of $E_{3}$ is

$$
\left(\lambda^{2}-\left(n_{1}+n_{2}\right) \lambda+\left(n_{1} n_{2}-n_{3} n_{4}\right)\right)\left(n_{5}-\lambda\right)\left(n_{6}-\lambda\right)=0,
$$

with

$$
\begin{aligned}
& n_{1}=r\left(1-\frac{S^{(3)}}{K}\right)-\frac{r S^{(3)}}{K}-p_{1}\left(1-m_{3}\right) Y_{S}^{(3)}-a_{1}, \\
& n_{2}=a_{3}\left(1-m_{3}\right) S^{(3)}-d_{3}, \\
& n_{3}=-p_{1}\left(1-m_{3}\right) S^{(3)}, \\
& n_{4}=a_{3}\left(1-m_{3}\right) Y_{S}^{(3)}, \\
& n_{5}=\beta S^{(3)}-p_{2}\left(1-m_{4}\right) Y_{S}^{(3)}-a_{2}, \\
& n_{6}=\gamma Y_{S}^{(3)}-a_{6},
\end{aligned}
$$

which has eigenvalues $\lambda_{1,2}=\frac{\left(n_{1}+n_{2}\right) \pm \sqrt{\left(n_{1}+n_{2}\right)^{2}-4\left(n_{1} n_{2}-n_{3} n_{4}\right)}}{2} ; \lambda_{3}=n_{5} ; \lambda_{4}=n_{6}$. So, we can say that the point $E_{3}$ is locally asymptotically stable if only if $n_{1}+n_{2}<0$; $n_{1} n_{2}>n_{3} n_{4} ; \beta S^{(3)}-p_{2}\left(1-m_{4}\right) Y_{S}^{(3)}<a_{2}$ and $\gamma Y_{S}^{(3)}<a_{6}$. On point $E_{4}$, the characteristic equation of $E_{4}$ is

$$
\left(z_{1}-\lambda\right)\left(\lambda^{3}+\varphi_{1} \lambda^{2}+\varphi_{2} \lambda+\varphi_{3}\right)=0 .
$$

One eigenvalue is $\lambda_{1}=z_{1}$, where $z_{1}=a_{3}\left(1-m_{3}\right) S^{(4)}+a_{4}\left(1-m_{4}\right) I^{(4)}-\gamma Y_{I}^{(4)}-d_{3}$. Other eigenvalues are obtained from the roots of cubic equations, which are obtained from the matrix $\hat{J}\left(E_{4}\right)$. 


$$
\hat{J}\left(E_{4}\right)=\left(\begin{array}{ccc}
z_{2} & z_{3} & 0 \\
\frac{\beta I^{(4)}}{1+I^{(4)}} & z_{4} & -p_{3}\left(1-m_{4}\right) I^{(4)} \\
0 & a_{3}\left(1-m_{4}\right) Y_{I}^{(4)} & a_{5}\left(1-m_{4}\right) I^{(4)}-a_{6}
\end{array}\right),
$$

with

$$
\begin{aligned}
& z_{2}=r\left(1-\frac{S^{(4)}+I^{(4)}}{K}\right)-\frac{r S^{(4)}}{K}-\frac{\beta I^{(4)}}{1+I^{(4)}}-a_{1}, \\
& z_{3}=-\frac{r S^{(4)}}{K}-\frac{\beta S^{(4)}}{1+I^{(4)}}+\frac{\beta S^{(4)} I^{(4)}}{\left(1+I^{(4)}\right)^{2}} \\
& z_{4}=\frac{\beta S^{(4)}}{1+I^{(4)}}-\frac{\beta S^{(4)} I^{(4)}}{\left(1+I^{(4)}\right)^{2}}-p_{3}\left(1-m_{4}\right) Y_{I}^{(4)}-a_{2} .
\end{aligned}
$$

Thus, the roots of the cubic equation are $\varphi_{1}=-\operatorname{tr}\left(\hat{J}\left(E_{4}\right)\right) ; \varphi_{2}=M_{11}+M_{22}+M_{33}$; and $\varphi_{3}=-\operatorname{det}\left(\hat{J}\left(E_{4}\right)\right)$, where $M_{i j}$ is minor of entry $\left(\hat{J}\left(E_{4}\right)\right)_{i j}$. By using Routh-Hurwitz criteria, the point $E_{4}$ is the locally asymptotically stable which can be provided with the following condition $\varphi_{1}>0 ; \varphi_{3}>0 ; \varphi_{1} \varphi_{2}>\varphi_{3} ; a_{3}\left(1-m_{3}\right) S^{(4)}+a_{4}\left(1-m_{4}\right) I^{(4)}<$ $\gamma Y_{I}^{(4)}+d_{3}$. On point $E_{5}$, the characteristic equation of $E_{5}$ is

$$
\left(e_{1}-\lambda\right)\left(\lambda^{3}+\delta_{1} \lambda^{2}+\delta_{2} \lambda+\delta_{3}\right)=0 .
$$

One eigenvalue is $\lambda_{1}=e_{1}$, where $e_{1}=\beta S^{(5)}-p_{2}\left(1-m_{4}\right) Y_{S}^{(5)}-p_{3}\left(1-m_{4}\right) Y_{I}^{(5)}-a_{2}$. Meanwhile, three eigenvalues are the roots of cubic equations $\lambda^{3}+\delta_{1} \lambda^{2}+\delta_{2} \lambda+\delta_{3}=0$, which are given by matrix $\hat{J}\left(E_{5}\right)$.

$$
\hat{J}\left(E_{5}\right)=\left(\begin{array}{ccc}
e_{2} & -p_{1}\left(1-m_{3}\right) S^{(5)} & 0 \\
a_{3}\left(1-m_{3}\right) Y_{S}^{(5)} & e_{3} & -\gamma Y_{S}^{(5)} \\
0 & \gamma Y_{I}^{(5)} & e_{4}
\end{array}\right),
$$

where

$$
\begin{aligned}
& e_{2}=r\left(1-\frac{S^{(5)}}{K}\right)-\frac{r S^{(5)}}{K}-p_{1}\left(1-m_{3}\right) Y_{S}^{(5)}-a_{1}, \\
& e_{3}=a_{3}\left(1-m_{3}\right) S^{(5)}-\gamma Y_{I}^{(5)}-d_{3}, \\
& e_{4}=\gamma Y_{S}^{(5)}-a_{6} .
\end{aligned}
$$

So, we obtain the roots of the cubic equation, i.e. $\delta_{1}=-\operatorname{tr}\left(\hat{J}\left(E_{5}\right)\right) ; \delta_{2}=M_{11}+M_{22}+$ $M_{33} ;$ and $\delta_{3}=-\operatorname{det}\left(\hat{J}\left(E_{5}\right)\right)$ with $M_{i j}$ is minor of entry $\left(\hat{J}\left(E_{5}\right)\right)_{i j}$. By using RouthHurwitz criteria, the point $E_{5}$ can be said locally asymptotically stable if it satisfies the following condition, $\beta S^{(5)}<p_{2}\left(1-m_{4}\right) Y_{S}^{(5)}+p_{3}\left(1-m_{4}\right) Y_{I}^{(5)}+a_{2} ; \delta_{1}>0 ; \delta_{3}>$ $0 ; \delta_{1} \delta_{2}>\delta_{3}$. On point $E_{6}$, the characteristic equation of $E_{6}$ is

$$
\left(l_{1}-\lambda\right)\left(\lambda^{3}+\sigma_{1} \lambda^{2}+\sigma_{2} \lambda+\sigma_{3}\right)=0 .
$$

One eigenvalue is $\lambda_{1}=l_{1}$, with $l_{1}=a_{5}\left(1-m_{4}\right) I^{(6)}+\gamma Y_{S}^{(6)}-a_{6}$. Three eigenvalues are the roots of cubic equations, i.e. $\lambda^{3}+\sigma_{1} \lambda^{2}+\sigma_{2} \lambda+\sigma_{3}=0$, which are given by matrix 
$\hat{J}\left(E_{6}\right)$.

$$
\hat{J}\left(E_{6}\right)=\left(\begin{array}{ccc}
l_{2} & l_{3} & -p_{1}\left(1-m_{3}\right) S^{(6)} \\
\frac{\beta I^{(6)}}{1+I^{(6)}} & l_{4} & -p_{2}\left(1-m_{4}\right) I^{(6)} \\
a_{3}\left(1-m_{3}\right) Y_{S}^{(6)} & a_{4}\left(1-m_{4}\right) Y_{S}^{(6)} & l_{5}
\end{array}\right),
$$

where

$$
\begin{aligned}
& l_{2}=r\left(1-\frac{S^{(6)}+I^{(6)}}{K}\right)-\frac{r S^{(6)}}{K}-\frac{\beta I^{(6)}}{1+I^{(6)}}-p_{1}\left(1-m_{3}\right) Y_{S}^{(6)}-a_{1}, \\
& l_{3}=-\frac{r S^{(6)}}{K}-\frac{\beta S^{(6)}}{1+I^{(6)}}+\frac{\beta S^{(6)} I^{(6)}}{\left(1+I^{(6)}\right)^{2}}, \\
& l_{4}=\frac{\beta S^{(6)}}{1+I^{(6)}}-\frac{\beta S^{(6)} I^{(6)}}{\left(1+I^{(6)}\right)^{2}}-p_{2}\left(1-m_{4}\right) Y_{S}^{(6)}-a_{2}, \\
& l_{5}=a_{3}\left(1-m_{3}\right) S^{(6)}+a_{4}\left(1-m_{4}\right) I^{(6)}-d_{3} .
\end{aligned}
$$

Thus, we obtained other corresponding eigenvalues, namely $\sigma_{1}=-\operatorname{tr}\left(\hat{J}\left(E_{6}\right)\right)$; $\sigma_{2}=M_{11}+M_{22}+M_{33} ; \sigma_{3}=-\operatorname{det}\left(\hat{J}\left(E_{6}\right)\right)$, with a minor of entry $\left(\hat{J}\left(E_{6}\right)\right)_{i j}$ that is denoted by $M_{i j}$. By using Routh-Hurwitz criteria, the point $E_{6}$ can be said locally asymptotically stable if only if $a_{5}\left(1-m_{4}\right) I^{(6)}+\gamma Y_{S}^{(6)}<a_{6} ; \sigma_{1}>0 ; \sigma_{3}>0 ; \sigma_{1} \sigma_{2}>\sigma_{3}$. On point $E_{7}$, we have the characteristic equation of $E_{7}$, which is obtained from the matrix $J^{*}\left(E_{7}\right)$. Meanwhile, $J^{*}\left(E_{7}\right)$ is the Jacobian matrix at $E_{7}$. Eigenvalues of $E_{7}$ are the roots of the fourth-order equation, i.e.

$$
\lambda^{4}+\eta_{1} \lambda^{3}+\eta_{2} \lambda^{2}+\eta_{3} \lambda+\eta_{4}=0
$$

with

$$
\begin{aligned}
& \eta_{1}=-\operatorname{tr}\left(J^{*}\left(E_{7}\right)\right) \\
& \eta_{2}=\tau_{11}+\tau_{22}+\tau_{33}+\varepsilon_{11}+\varepsilon_{22}+\rho_{11}, \\
& \eta_{4}=-\left(M_{11}+M_{22}+M_{33}+M_{44}\right) \\
& \eta_{4}=\operatorname{det}\left(J^{*}\left(E_{7}\right)\right)
\end{aligned}
$$

where $\tau(\varepsilon)$ and $\rho$ are minor of order 2 from the remaining submatrix after $4^{\text {th }}$ row and $4^{\text {th }}$ column (after $3^{\text {rd }}$ row and $3^{\text {rd }}$ column) and after $2^{\text {nd }}$ row and $2^{\text {nd }}$ column are removed from $J^{*}\left(E_{7}\right)$, respectively. Meanwhile, $M_{i j}$ is minor of order 3 for entry $\left(J^{*}\left(E_{7}\right)\right)_{i j}$. By using Routh-Hurwitz criteria, we get the condition for the stability of asymptotical locally at the point $E_{7}$, namely $\eta_{1}>0 ; \eta_{1} \eta_{2}>\eta_{3} ; \eta_{3}\left(\eta_{2} \eta_{1}-\eta_{3}\right)-\eta_{4} \eta_{1}^{2}>0 ; \eta_{4}>0$.

\subsection{Numerical Simulation}

In this section, we present some numerical solutions by using the Runge-Kutta $4^{\text {th }}$ order as a numerical method to illustrate our analytical results. We use some parameters for the system (1), namely $r=0.45, K=10, \gamma=0.15, p_{1}=0.6, p_{2}=0.8, p_{3}=$ $0.45, a_{1}=0.12, a_{2}=0.16, a_{3}=0.25, a_{4}=0.35, a_{5}=0.15, a_{6}=0.16, d_{3}=$ $0.103, \beta=0.3333$.

Case 1: We start with $m_{3}=0.157$ and $m_{4}=0.108$. The prey refuge is very small, which means that the number of prey outside the refuge is huge. All solutions are convergent to 
$E_{3}(0.4887,0,0.6090,0)$, see figure 2 (a). This equilibrium is also locally asymptotically stable, see figure 2(b). It indicates that infected predator and infected prey are extinct.

Case 2: We consider $m_{3}=0.65$ and $m_{4}=0.3$. The prey refuge means that the number of prey outside prey refuge is less than the first case. All solutions with different values are convergent to equilibrium $E_{5}(2.3556,0,1.0667,0.6874)$, see figure $3(\mathrm{a}) . E_{5}$ is also locally asymptotically stable, see figure 3(b). It indicates that the infected prey is extinct.

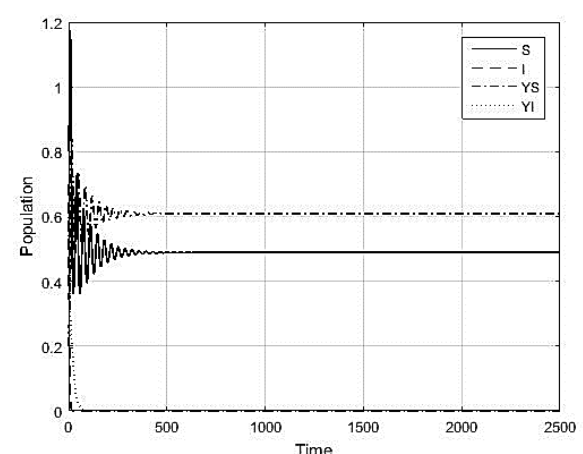

(a)

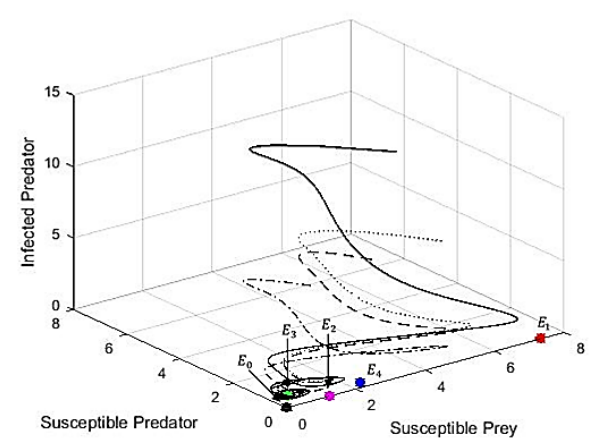

(b)

Figure 2. Numerical solution for case 1: (a) time graph; (b) the phase portrait

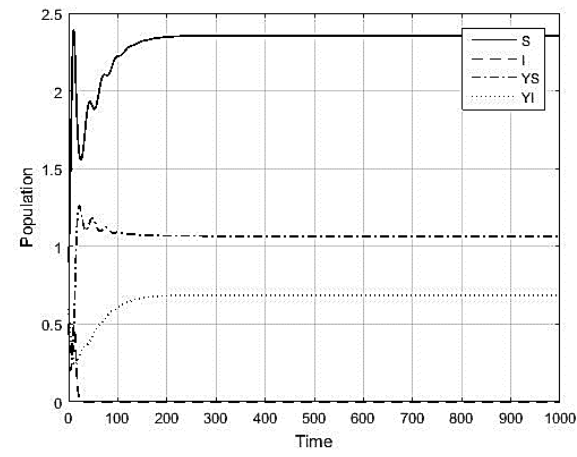

(a)

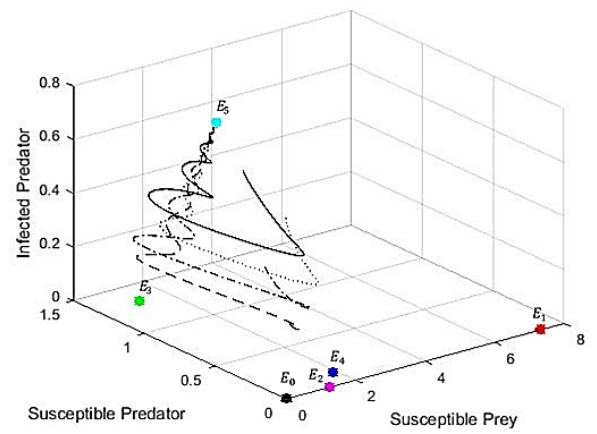

(b)

Figure 3. Numerical solution for case 2: (a) time graph; (b) the phase portrait

Case 3: When the prey refuge increases to become $m_{3}=0.85$ and $m_{4}=0.5$, then it shows that amount of prey outside refuge is small. All trajectories with different values converge to the point of equilibrium $E_{7}(2.7641,0.4901,0.8216,0.5761)$, see figure $4(a)$, and is also locally asymptotically stable, see figure 4(b). It indicates that all populations exist.

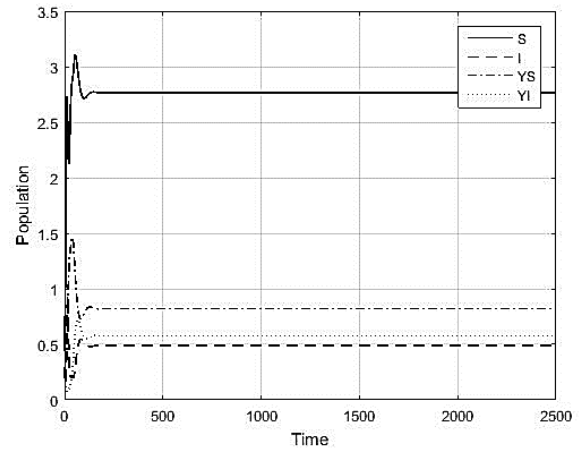

(a)

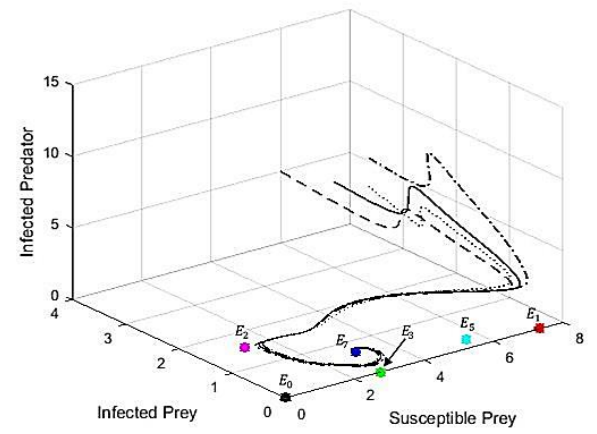

(b)

Figure 4. Numerical solution for case 3: (a) time graph; (b) the phase portrait

To investigate the effect of refuge in prey populations, we can observe the dynamic behavior of the system (1) by using $m_{3}, m_{4}$ makes different values. Figure 5 shows the 
time graph of prey populations which means that prey refuge can prevent extinction in prey populations when $m_{3}, m_{4}$ values are getting large.
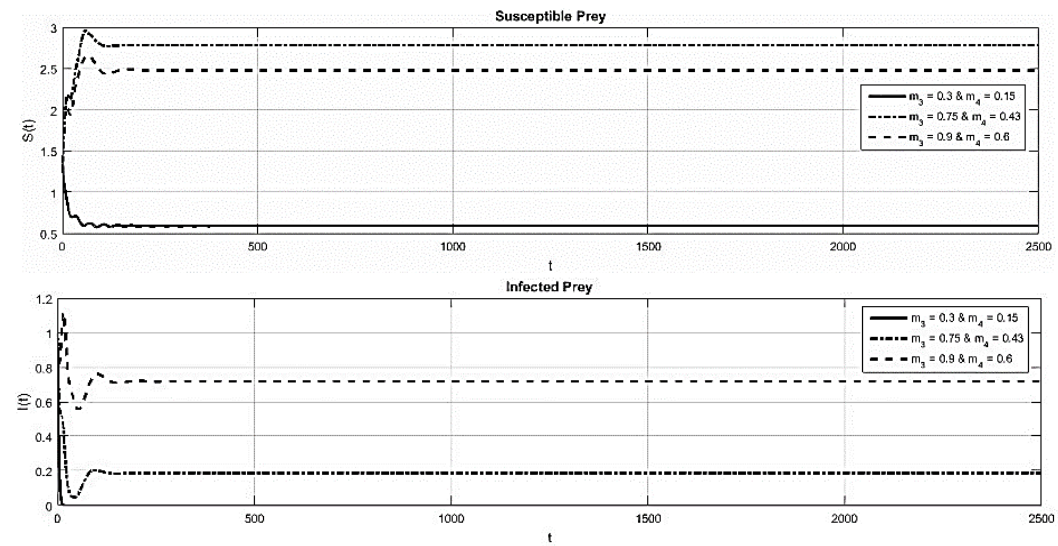

Figure 5. Influence refuge in prey by using $m_{3}, m_{4}$ makes different values

\section{Conclusions}

In this paper, we have merged an eco-epidemiology model with transmission disease in both populations by using non-linear incidence rate in prey populations and also prey refuge proportional in prey populations. Eight equilibrium points that exist under certain conditions and also that local stability for those points have been determined. We have noticed that prey refuge can avoid extinction in prey populations. For future work, we have to investigate the harvesting effect in both populations. It is used as a disease control in an eco-epidemiology model.

\section{References}

[1] W. O. Kermack and A. G. McKendrick, "A Contribution to the Mathematical Theory of Epidemics," Proc. R. Soc. London, vol. 115, no. 772, p. 22, 1927.

[2] R. M. Anderson and R. M. May, "The invasion, persistence and spread of infectious diseases within animal and plant communities," Philos. Trans. R. Soc. Lond. B. Biol. Sci., vol. 314, no. 1167, pp. 533-570, 1986, doi: 10.1098/rstb.1986.0072.

[3] C. Maji, D. Kesh, and D. Mukherjee, "Bifurcation and global stability in an ecoepidemic model with refuge," Energy, Ecol. Environ., vol. 4, no. 3, pp. 103-115, 2019, doi: 10.1007/s40974-019-00117-6.

[4] S. Kant and V. Kumar, "Analysis of an eco-epidemiological model with migrating and refuging prey," Springer Proc. Math. Stat., vol. 143, pp. 17-36, 2015, doi: 10.1007/978-81-322-2485-3_2.

[5] A. K. Pal and G. P. Samanta, "Stability analysis of an eco-epidemiological model incorporating a prey refuge," Nonlinear Anal. Model. Control, vol. 15, no. 4, pp. 473491, 2010, doi: 10.15388/na.15.4.14319.

[6] S. A. Wuhaib and Y. A. B. U. Hasan, "Predator-Prey Interactions With Harvesting of Predator With Prey in Refuge," Commun. Math. Biol. Neurosci., vol. 2013, no. 1, pp. 1-19, 2013.

[7] S. P. Bera, A. Maiti, and G. P. Samanta, "A prey-predator model with infection in both prey and predator," Filomat, vol. 29, no. 8, pp. 1753-1767, 2015, doi: 10.2298/FIL1508753B. 
[8] S. Kant and V. Kumar, "Stability analysis of predator-prey system with migrating prey and disease infection in both species," Appl. Math. Model., vol. 42, pp. 509539, 2017, doi: 10.1016/j.apm.2016.10.003.

[9] A. Pusparani, W. M. Kusumawinahyu, and Trisilowati, "Dynamical Analysis of Infected Predator-Prey Model with Saturated Incidence Rate," IOP Conf. Ser. Mater. Sci. Eng., vol. 546, no. 5, p. 8, 2019, doi: 10.1088/1757-899X/546/5/052055.

[10] V. Capasso and G. Serio, "A generalization of the Kermack-McKendrick deterministic epidemic model," Math. Biosci., vol. 42, no. 1-2, pp. 43-61, 1978, doi: 10.1016/0025-5564(78)90006-8.

[11] W. min Liu, S. A. Levin, and Y. Iwasa, "Influence of nonlinear incidence rates upon the behavior of SIRS epidemiological models," J. Math. Biol., vol. 23, no. 2, pp. 187204, 1986, doi: 10.1007/BF00276956.

[12] S. Ruan and W. Wang, "Dynamical behavior of an epidemic model with a nonlinear incidence rate," J. Differ. Equ., vol. 188, no. 1, pp. 135-163, 2003, doi: 10.1016/S0022-0396(02)00089-X.

[13] R. K. Naji and A. N. Mustafa, "The dynamics of an eco-epidemiological model with nonlinear incidence rate," J. Appl. Math., vol. 2012, p. 24, 2012, doi: $10.1155 / 2012 / 852631$.

[14] A. P. Maiti, C. Jana, and D. K. Maiti, "A delayed eco-epidemiological model with nonlinear incidence rate and Crowley-Martin functional response for infected prey and predator," Nonlinear Dyn., vol. 98, no. 2, pp. 1137-1167, 2019, doi: 10.1007/s11071-019-05253-6.

[15] S. Sharma and G. P. Samanta, "A Leslie-Gower predator-prey model with disease in prey incorporating a prey refuge," Chaos, Solitons and Fractals, vol. 70, no. 1, pp. 69-84, 2015, doi: 10.1016/j.chaos.2014.11.010.

[16] S. Wang, Z. Ma, and W. Wang, "Dynamical behavior of a generalized ecoepidemiological system with prey refuge," Adv. Differ. Equations, vol. 2018, no. 1, pp. 1-20, 2018, doi: 10.1186/s13662-018-1704-x.

[17] M. Onana, B. Mewoli, and J. J. Tewa, "Hopf bifurcation analysis in a delayed LeslieGower predator-prey model incorporating additional food for predators, refuge and threshold harvesting of preys," Nonlinear Dyn., vol. 100, no. 3, pp. 3007-3028, 2020, doi: 10.1007/s11071-020-05659-7.

[18] Y. Cai, C. Zhao, W. Wang, and J. Wang, "Dynamics of a Leslie-Gower predatorprey model with additive Allee effect," Appl. Math. Model., vol. 39, no. 7, pp. 20922106, 2015, doi: 10.1016/j.apm.2014.09.038. 